2. W. Benz, Ueber Möbiusebenen. Ein Bericht. Jber. Deutsch. Math.-Verein. 63 (1960), 1-27.

3. P. Dembowski and A. Wagner, Some characterizations of finite projective spaces, Arch. Math. 11 (1960), 465-469.

4. G. Ewald, Beispiel einer Möbiusebene mit nichtisomorphen affinen Unterebenen, Arch. Math. 11 (1960), 146-150.

5. A. J. Hoffman, Chains in the projective line, Duke Math. J. 18 (1951), 827-830.

6. D. R. Hughes, Combinatorial analysis. t-designs and permutation groups, 1960 Institute on Finite Groups, Proc. Sympos. Pure Math. Vol. 6, pp. 39-41, Amer. Math. Soc., Providence, R. I., 1962.

7. B. Qvist, Some remarks concerning curves of the second degree in a finite plane, Ann. Acad. Sci. Fenn. 134 (1952).

8. B. Segre, On complete caps and ovaloids in three-dimensional Galois spaces of characteristic two, Acta Arith. 5 (1959), 315-332.

9. J. Tits, Ovoïdes a translations, Rend. Mat. e Appl. 21 (1962), 37-59.

10. - - Ovoïdes et groupes de Suzuki, Arch. Math. 13 (1962), 187-198.

11. B. L. van der Waerden und L. J. Smid, Eine Axiomatik der Kreisgeometrie und der Laguerregeometrie, Math. Ann. 110 (1935), 753-776.

12. A. Winternitz, Zur Begründung der projektiven Geometrie. Einfïhrung idealer Elemente, unabhängig von der Anordnung, Ann. of Math. (2) 41 (1940), 365-390.

UNIVERSITÄT FRANKFURT AM MAIN AND

Queen Mary College, London

\title{
MICROBUNDLES ARE FIBRE BUNDLES ${ }^{1}$
}

\author{
BY J. M. KISTER
}

Communicated by Deane Montgomery, June 10, 1963

Introduction. In [1], Milnor develops a theory for structures, known as microbundles which generalize vector bundles. It is shown there that this is a proper generalization; that some microbundles cannot be derived from any vector bundle. It is then possible, for instance, to find a substitute (tangent microbundle) for the tangent bundle over a manifold $M$ even though $M$ admits no differential structure.

A well-known and more general class of structures than vector bundles (but less general than microbundles) is the class of fibre bundles with a Euclidean fibre and structural group the originpreserving homeomorphisms of Euclidean space topologized by the compact-open topology (cf. [2]). In this note such structures will be

1 This work was supported by a grant from the Institute for Advanced Study and by NSF grant G-24156. 
denoted simply as bundles.

Briefly then, our main result is that every microbundle over a complex is isomorphic to a bundle, in fact it contains a bundle and that this bundle is unique. The same result for microbundles over manifolds and, more generally, ANR's in Euclidean space follows easily. In the case of the tangent microbundle over a topological manifold $M$ this means that for each point $x$ in $M$ there is selected a neighborhood $U_{x}$ which is homeomorphic to Euclidean space and $U_{x}$ varies continuously with $x$. In the case of a differentiable manifold this selection is accomplished by means of a Riemannian metric. It was the absence of such a selection, however, that provided impetus to the consideration of microbundles (cf. Introduction in [1]).

I wish to express my gratitude to D. R. McMillan for several profitable conversations.

Statement of results. Let $R^{l}$ denote $l$-dimensional Euclidean space and $\mathcal{G}$ the space of all imbeddings of $R^{l}$ into $R^{l}$ provided with the compact-open topology. Let $g_{0}$ be the origin-preserving elements of $\mathcal{G}$, $\mathfrak{H}$ those elements of $\mathcal{G}$ whose images are $R^{l}$, and $\mathfrak{H C}_{0}=\mathcal{G}_{0} \cap \mathcal{H}$.

A microbundle $X$, having fibre dimension $l, B \rightarrow^{i} E \rightarrow^{i} B$, admits a bundle providing there is an open set $E_{1}$ in $E$ containing the 0 -section $i(B)$ such that $j \mid E_{1}: E_{1} \rightarrow B$ is a fibre bundle with fibre $R^{l}$ and structural group $\nVdash_{0}$. The fibre bundle in this case will be called an admissible bundle.

Let $T_{n}$ be the statement that every microbundle over a locallyfinite $n$-dimensional complex admits a bundle. Let $U_{n}$ be the statement that any two admissible bundles for the same microbundle over a locally-finite $n$-dimensional complex are isomorphic. An isomorphism in this case is a homeomorphism between the total spaces preserving fibres and which is the identity on the 0 -section.

THEOREM. $T_{n}$ and $U_{n}$ are true for all $n$.

The proof will be sketched, with details appearing in a later paper. $T_{0}$ and $U_{0}$ follow immediately from the fact that microbundles over a 0 -dimensional set are all trivial. We finish the proof by showing

(1) $T_{n-1}$ and $U_{n-1}$ imply $T_{n}$, and

(2) $T_{n}$ implies $U_{n}$.

The following lemma is used repeatedly.

LEMma. There is a map $F: \mathcal{G}_{0} \times I \rightarrow G_{0}$ such that

(a) $F(g, 0)=g$, all $g \in G_{0}$,

(b) $F(g, 1) \in \mathcal{H C}_{0}$, all $g \in \mathcal{G}_{0}$,

(c) $F(h, t) \in \mathcal{F}_{0}$, all $h \in \mathcal{H}_{0}, t \in I$. 
The proof for the Lemma consists of analyzing the isotopy, which is relatively easy to produce, taking a single element in $\mathcal{G}_{0}$ into $\mathcal{H}_{0}$ and expressing the process in a canonical fashion. Then one has to verify that this process yields nearby isotopies if the elements in $g_{0}$ are close in the compact-open topology. The full verification is long and somewhat tedious and is therefore suppressed here.

We return to the proof of (1). Let $X$ be a microbundle over a locally-finite $n$-complex $K$ with diagram: $K \rightarrow^{i} E \rightarrow^{i} K$. For each $n$ simplex $\sigma$ in $K$ we find an admissible trivial bundle $\xi_{\sigma}$ for $X \mid \sigma$. Next let $D$ be an open set in $E$ containing $i(K)$ such that $j^{-1}(\sigma) \cap D \subset E\left(\xi_{\sigma}\right)$ the total space of $\xi_{\sigma}$. Let $K^{n-1}$ denote the $(n-1)$-skeleton of $K$ and $Y$ the microbundle $K^{n-1} \rightarrow^{i^{\prime}} j^{-1}\left(K^{n-1}\right) \cap D \rightarrow^{j^{\prime}} K^{n-1}$, where $i^{\prime}$ and $j^{\prime}$ are the restrictions of $i$ and $j$. By $T_{n-1}, Y$ admits a bundle $\eta$, and by the choice of $D$, for each point $z$ in $\partial \sigma$, the $\eta$-fibre over $z$ is contained in the $\xi_{\sigma}$-fibre over $z . \eta \mid \partial \sigma$ and $\xi_{\sigma} \mid \partial \sigma$ are admissible bundles for $X \mid \partial \sigma$ and since the second is trivial, by $U_{n-1}$ it follows that $\eta \mid \partial \sigma$ is trivial also. This permits us to coordinatize both the $\eta$-fibres and $\xi_{\sigma}$-fibres over $\partial \sigma$. The inclusion of the former in the latter determines a map of $\partial \sigma$ into $S_{0}$ which by the Lemma can be deformed into a map of $\partial \sigma$ into $\mathcal{H}_{0}$. If $\sigma_{1}$ is a smaller concentric simplex in $\sigma$ we may regard this deformation as assigning elements in $\mathcal{G}_{0}$ to points in $\sigma$-int $\sigma_{1}$ so that the elements assigned to points in $\partial \sigma_{1}$ are all in $\mathcal{H}_{0}$. This correspondence permits us to smooth the fibres in going from the $\eta$ fibres over $\partial \sigma$ to the $\xi_{\sigma}$-fibres over $\sigma_{1}$. By repeating this process on each $n$-simplex $\sigma$ the bundle $\eta$ over $K^{n-1}$ can be extended to a bundle over $K$.

To prove (2) let $\sigma_{1}, \sigma_{2}, \cdots, \sigma_{\alpha}, \cdots$ be a well-ordering of those simplexes in the $n$-complex $K$ which are not faces of some higher dimensional simplex in $K$. Let $\xi_{1}$ and $\xi_{2}$ be two admissible bundles for $X$, a microbundle over $K$. By $T_{n}$ there is no loss in generality in assuming $E\left(\xi_{1}\right) \subset E\left(\xi_{2}\right)$. Let $f_{0}: E\left(\xi_{1}\right) \rightarrow E\left(\xi_{2}\right)$ be the inclusion. Let $N\left(\sigma_{\alpha}\right)$ be the closed star neighborhood of $\sigma_{\alpha}$ in the second barycentric subdivision. Let $K_{\alpha}=\bigcup_{\beta \S \alpha} \sigma_{\beta}$, a subcomplex.

Suppose for each $\beta<\alpha$ we have defined $f_{\beta}: E\left(\xi_{1}\right) \rightarrow E\left(\xi_{2}\right)$, an imbedding, taking fibres into fibres, and $f_{\beta}$ is the identity on $i(K)$. Suppose further that $f_{\beta} \mid K_{\beta}$ is an isomorphism from $\xi_{1} \mid K_{\beta}$ onto $\xi_{2} \mid K_{\beta}$ and that for each point $p$ in $E\left(\xi_{1}\right)-j^{-1}\left(N\left(\sigma_{\beta}\right)\right)$ there is a $\gamma<\beta$ and a neighborhood $N$ of $p$ such that $f_{\beta}\left|N=f_{\beta^{\prime}}\right| N$ for $\gamma \leqq \beta^{\prime} \leqq \beta$. We construct $f_{\alpha}$ satisfying these properties.

Let $g_{\alpha}: E\left(\xi_{1}\right) \rightarrow E\left(\xi_{2}\right)$ be $f_{\alpha-1}$ if $\alpha-1$ exists. Otherwise $g_{\alpha}=\operatorname{limit}_{\beta \rightarrow \alpha} f_{\beta}$, which exists since each point in $K$ lies in only finitely-many $N\left(\sigma_{\beta}\right)$. Then $g_{\alpha}\left(E\left(\xi_{1}\right)\right)$ is the total space of a bundle $\eta_{\alpha}$ in a natural way. Since 
$N\left(\sigma_{\alpha}\right)$ is contractible $\eta_{\alpha} \mid N\left(\sigma_{\alpha}\right)$ and $\xi_{2} \mid N\left(\sigma_{\alpha}\right)$ are trivial. This allows us to coordinatize the two sets of fibres and the inclusion of the $\eta_{\alpha}$-fibres in the $\xi_{2}$-fibres assigns an element $g^{z}$ in $\oint_{0}$ to every $z$ in $N\left(\sigma_{\alpha}\right)$. Let $t: K \rightarrow I$ be a map such that $t\left(\sigma_{\alpha}\right)=1$ and $t\left(K-N\left(\sigma_{\alpha}\right)\right)=0$. Using the Lemma we associate with each $z$ in $N\left(\sigma_{\alpha}\right)$ the imbedding $h^{z}=F\left(g^{z}, t(z)\right)$. Then for each $z$ in $\sigma_{\alpha}, h^{z}$ is in $\mathcal{F}_{0}$, and for $z$ in $\mathrm{Cl}\left(K-N\left(\sigma_{\alpha}\right)\right) \cap N\left(\sigma_{\alpha}\right), h^{z}=g^{z}$. If we define a homeomorphism $h_{\alpha}$ from $E\left(\eta_{\alpha}\right)$ to $E\left(\xi_{2}\right)$ by taking the $\eta_{\alpha}$-fibre over $z$ into the $\xi_{2}$-fibre over $z$ according to $h^{z}$, for each $z$ in $N\left(\sigma_{\alpha}\right)$ and using the inclusion elsewhere, then $f_{\alpha}=h_{\alpha} g_{\alpha}$ satisfies the desired properties of the induction.

The isomorphism from $\xi_{1}$ onto $\xi_{2}$ is the limit of the $f_{\alpha}$. This proves (2) and finishes the proof of the Theorem.

COROLlaRY 1. If $B$ is a neighborhood retract in some Euclidean space, for example a manifold, then any microbundle over $B$ admits a unique bundle.

Proof. Let $B$ be a subset of $R^{n}$ and $V$ an open set in $R^{n}$ containing $B$ and $\rho: V \rightarrow B$, a retraction. Then if $X$ is a microbundle over $B$, $\rho^{*}(X)$ may be regarded as an extension of $X$ to all of $V$. But $V$ can be triangulated and the Theorem applied to give both the existence and uniqueness.

Let $\mathfrak{F C}_{0}^{+}(n)$ be the orientation-preserving, origin-preserving homeomorphisms of $R^{n}$ onto $R^{n}$. As a consequence of the Theorem and the fact that $k_{0} S^{8} \rightarrow k_{\text {top }} S^{8}$ is not an isomorphism [1], we have:

COROLlaRy 2. For large enough $n$, the homomorphism $\pi_{7}(\mathrm{SO}(n))$ $\rightarrow \pi_{7}\left(\mathcal{H}_{0}^{+}(n)\right)$ induced by inclusion is not an isomorphism.

Added in proof. The author has learned that B. Mazur has obtained independently a somewhat different proof of the Theorem.

\section{REFERENCES}

1. J. Milnor, Microbundles, Part I, Princeton University, 1963 (mimeographed).

2. N. Steenrod, The topology of fibre bundles, Princeton Univ. Press, Princeton, N. J., 1951.

INSTITUTE FOR AdVANCED STUdy 